\title{
3 Research Suare

\section{Difference in autonomic nervous effect of blue light depending on the angle of incidence on the eye}

\section{Emi Yuda}

Tohoku Daigaku - Aobayama Shin Campus

\section{Yutaka Yoshida}

Nagoya Shiritsu Daigaku

Norihiro Ueda

Nagoya Shiritsu Daigaku

Junichiro Hayano ( $\nabla$ hayano@med.nagoya-cu.ac.jp )

Nagoya City University https://orcid.org/0000-0002-5340-6325

\section{Research note}

Keywords: autonomic nervous system, blue light, intrinsically-photosensitive retinal ganglion cell, lightemitting diode, non-image forming function, smartphone, solid-state lighting

Posted Date: January 10th, 2020

DOI: https://doi.org/10.21203/rs.2.20527/v1

License: (c) (1) This work is licensed under a Creative Commons Attribution 4.0 International License. Read Full License

Version of Record: A version of this preprint was published at BMC Research Notes on March 10th, 2020. See the published version at https://doi.org/10.1186/s13104-020-04988-5. 


\section{Abstract}

Objective: Blue light has been attributed to the adverse biological effects caused by the use of smartphones and tablet devices at night. However, it is not realistic to immediately avoid nighttime exposure to blue light in the lifestyle of modern society, so other effective methods to reduce the unwanted influences should be investigated. Earlier studies reported that inferior retinal light exposure causes greater melatonin suppression than superior retinal exposure. We examined whether the autonomic responses to blue light depends on the angle of incidence on the eye.

Results: In eight healthy subjects, blue light from organic electroluminescent lighting device (15.4 Ix at subjects' eye) was exposed from 6 angles $\left(0^{\circ}, 30^{\circ}, 45^{\circ}, 135^{\circ}, 150^{\circ}\right.$, and $\left.180^{\circ}\right)$ for 5 min each with a $10-$ min interval of darkness. After adjusting the order effect of angles, however, no significant difference was detected in autonomic indices of heart rate variability with the angle of incidence, except that the exposure from $45^{\circ}$ caused lower heart rate than exposures from $0^{\circ}, 150^{\circ}$, and $180^{\circ}$. Further researches on the physiological properties of the biological effects of blue light are needed to control and reduce the health risks of using blue-light-emitting devices.

\section{Introduction}

While light is an image forming signal for vision, light reaching the retina also causes various non-image forming reactions, such as pupil size adjustment, increased vigilance, autonomic nervous arousal, and circadian clock adaptation [1]. These non-image forming functions were known to be mediated by intrinsically photosensitive retinal ganglion cells (ipRGCs) [2, 3], which express melanopsin as the photopigment. Since melanopsin has a specific sensitivity to blue wavelength light around $480 \mathrm{~nm}$ [4], blue light stimulation to the ipRGCs is recognized as a central mediator of the non-image-forming response to light [5-7].

In recent years, blue light has been attributed to the adverse biological effects caused by the use of smartphones and tablet devices at night [8-11]. Accumulating evidence shows that the use of these lightemitting devices immediately before bedtime has negative impact on sleep $[12,13]$. The displays of these devices use solid-state lighting (SSL), most of which emits much blue light even though they appear to emit white light $[10,11]$.

SSLs, however, have many benefits over traditional light sources and they deeply penetrate into the lifestyle, education, and industry of modern societies that are operating $24 \mathrm{~h}$ of a day. It seems impractical to immediately avoid exposure to blue light at night. We therefore need to accumulate our knowledge to control the negative biological effects of blue light. Previous studies have suggested the influences of the distance to light source emitting blue light [14] and of the angle of incidence of light on the eye. Particularly, white light exposure to the inferior retina may cause greater melatonin suppression than exposure to the superior retina $[15,16]$. In this study, we examined whether the autonomic effects of blue light depend on the incidence angle on the eye. 


\section{Methods}

\section{Methods}

The present study was performed according to the protocol approved by the Ethics Review Committee of Nagoya City University Graduate School of Medical Sciences and Nagoya City University Hospital (No. 60160164).

\section{Subjects}

We studied eight subjects (mean age \pm SD, $22 \pm 3$ year, seven males and a female) who had normal color vision, were not taking any medications for $>2$ weeks, and displayed a normal sinus rhythm on electrocardiogram (ECG) at rest. All subjects gave their written informed consent to participate in this study.

\section{Lighting apparatus}

An organic electroluminescent (OEL) lighting device developed for research purposes was used. The device consisted of four OEL panels (VELVE OLED Lighting Module with adjustable RGB color and brightness, $55 \times 55$ mm square, Mitsubishi Chemical Pioneer OLED Lighting Corporation, Tokyo, Japan) that were linearly aligned with two panels at the both sides inclining inward with an angle of $40^{\circ}$. Using a custom-made experimental frame, the lighting device was secured at a constant distance of $24 \mathrm{~cm}$ from the eyes of subject lying on a bed in the supine position so that the four OEL panels were aligned across their body axis and able to be moved in their sagittal plane keeping the light axes always facing their eyes (Figure 1).

Although the device was able to emit red, green, and blue in any combinations, only $100 \%$ blue light was used in this study. Front illuminance were $15.4 \mathrm{~lx}$ at subject's eye position, chromaticity $(x, y)$ was $(0.14$, $0.16)$, and the melanopsin-stimulating component that was estimated from the melanoptic spectral efficiency curve adjusted for the effect of human pre-receptoral filtering [17-19] were $75 \%$ of the total photon flux density $\left(0.377 \mu \mathrm{mol} /\left[\mathrm{m}^{2} \cdot \mathrm{s}\right]\right)$.

\section{Study protocol}

Subjects were instructed not to consume food or beverages containing caffeine or alcohol after 21:00 the previous night. The experiments were performed between 10:00 and 17:00 in a calm, light-shielded, and air-conditioned $\left(24 \pm 2{ }^{\circ} \mathrm{C}\right)$ laboratory more than two hour after a light meal. Subjects were laid supine on a bed and instructed to continue to look at the mark on the ceiling just above their heads while they were exposed to light, and not to look directly the light source. After a dark adaptation for $10 \mathrm{~min}$, the blue light from the device was exposed for $5 \mathrm{~min}$ from each of six angles $\left(0^{\circ}, 30^{\circ}, 45^{\circ}, 135^{\circ}, 150^{\circ}\right.$, and $\left.180^{\circ}\right)$. The order of the angle was counterbalanced among subjects. These was a 10-min dark interval period after light exposure from each angle. 


\section{Measurement and data analysis}

During the experiment including light exposure and dark periods, ECG was recorded continuously with bipolar CM5 lead with a bioelectric amplifier (Biotop mini, East Medic Corporation, Kanazawa, Japan), digitized at $500 \mathrm{~Hz}$ with an analog-to-digital converter (AIO-163202FX-USB, CONTEC Corporation, Osaka, Japan), and stored in a hard disk.

From ECG signal, R-R interval time series were obtained and divided into segments of 5-min light exposure and 10-min dark periods for each incident angle. For each segment, mean heart rate, standard deviation of R-R interval (SDNN), and low-frequency (LF, 0.04-0.15 Hz) and the high-frequency (HF, 0.15$0.40 \mathrm{~Hz}$ ) components of heart rate variability (HRV) were computed.

\section{Statistical analysis}

Statistical Analyses System version 9.4 (SAS institute Inc., Cary, NC, USA) was used for the statistical analysis. The Mixed procedure was used for analysis of variance for repeated measures with incident angle, light-to-dark, and exposure order as the fixed effects and subject as the random effect. $P<0.05$ was considered to be statistically significant and Bonferroni adjustment was used to keep type 1 error level in multiple comparisons.

\section{Results}

Figure 2 shows heart rate and HRV indices during blue light exposure and subsequent dark period from each incidence angle. Data are least-square means adjusted for order effect. The statistical analysis for the effects of incident angle, light-to-dark, order, and interaction between the incident angle and light-todark was presented in Table 1. 
Table 1

Results of repeated ANOVA

\begin{tabular}{|llllllllll|}
\hline & \multicolumn{3}{l}{ Incident angle } & \multicolumn{2}{l}{ Light-to-dark } & Order & & \multicolumn{3}{l|}{ Interaction* $^{*}$} \\
& F & P & F & P & F & P & F & P \\
\hline Heart rate & 2.48 & 0.01 & 0.15 & 0.7 & 7.85 & 0.006 & 0.31 & 0.9 \\
\hline SDNN & 0.61 & 0.8 & 1.92 & 0.1 & 17.04 & $<.0001$ & 0.14 & 0.9 \\
\hline LF power & 0.3 & 0.9 & 1.49 & 0.2 & 18.89 & $<.0001$ & 0.2 & 0.9 \\
\hline HF power & 0.7 & 0.7 & 0.97 & 0.3 & 14.22 & 0.0003 & 0.12 & 0.9 \\
\hline LF/HF & 0.68 & 0.7 & 0.55 & 0.4 & 2.79 & 0.09 & 0.81 & 0.5 \\
\hline HF frequency & 1.14 & 0.3 & 1.47 & 0.2 & 2.77 & 0.1003 & 0.24 & 0.9 \\
\hline *Interaction between the incident angle and light-to-dark. & & & \\
\hline
\end{tabular}

The incident angle showed a significant effect on heart rate only $(P=0.01)$, but not on any HRV autonomic indices. There was no significant effect of light-to-dark or of interaction between the incident angle and light-to-dark. On the other hand, significant effects of exposure angle order were observed on heart rate, SDNN, and LF and HF power. Multiple comparison showed that heart rate for $45^{\circ}$ was lower than heart rate for $0^{\circ}, 150^{\circ}$, and $180^{\circ}$.

\section{Discussion}

To examine whether autonomic responses to blue light depend on the angle of incidence on the eye, we analyzed HRV during and after blue light exposures from various angles. However, no significant difference was detected in HRV autonomic indices with the angle of incidence, although the exposure from $45^{\circ}$ caused lower heart rate than exposures from $0^{\circ}, 150^{\circ}$, and $180^{\circ}$.

To our knowledge, this is the first study to analyze the effect of incidence angle on the non-image forming functions of blue light. As to white light, however, Lasko et al. [15] reported that nighttime bright white light in the upper visual field caused greater melatonin suppression than the light in the lower visual field. Similarly, Glickman et al. [16] demonstrated that nighttime white light exposure to the inferior retina caused melatonin suppression, while exposure to the superior retina caused no significant suppression. We have previously observed that exposure to OEL blue light causes a suppression of HF component of HRV, while red and green OEL light show no such effect [20]. These findings suggest that non-image forming effects of light including those on autonomic functions may differ with the incidence angle on the eye. These findings motived us to perform the present study, but no clear evidence supporting for the difference in the autonomic effect of blue light with the incidence angle was obtained. 
We observed that the heart rates during and after exposure from $45^{\circ}$ were lower than those from $0^{\circ}, 150^{\circ}$, and $180^{\circ}$. The mechanism of this effect is unclear, as no significant differences were observed in any autonomic index, including LF and HF power and LF-to-HF ratio. One may speculate, however, that the subjects were more relaxed because they might have been more accustomed to light from $45^{\circ}$ compared to lights from other angles in their daily life.

Although the present study failed to detect the significant effect of the incidence angle on autonomic responses to blue light, it seems important to expand our knowledge of biological effects of blue light. Many authors have warned the health risk of nighttime use of smartphones and tablet devices that emit blue light [8-12]. The displays of these devices use SSLs for the back light of liquid crystal, most of which are white light-emitting diodes (LEDs) made from a combination of blue LED at $460 \mathrm{~nm}$ and a broad-spectrum yellow garnet phosphor $[10,11]$. Even though they appear to emit white light, they emit much blue light that can stimulate melanopsin. Thus, the use of these device at night is thought to evoke adverse non-image forming responses through ipRGCs. Actually, Chang et al. [13] performed a study with a randomized, cross-over design to compare the effects of reading on an electric book emitting blue light

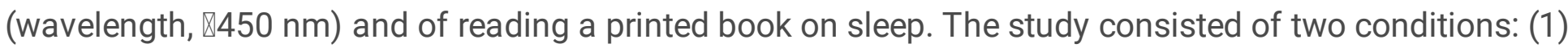
4-h of reading on a light-emitting electric book before bedtime in very dim room light for five consecutive evenings and (2) 4-h reading of a printed book in the same very dim light room before bedtime for five consecutive evening. They observed that, compared with the printed book condition, the electric book condition suppressed evening melatonin levels, delayed dim light melatonin onset, lengthened sleep latency, decreased evening sleepiness, and reduced next morning alertness. Although there are no studies to report the autonomic effect of the use of smartphones or tablet devices, the autonomic effect of blue light itself has been reported previously $[20,21]$. In a study of the autonomic neural effects of OEL light with various colors, we demonstrated a greater suppression of vagal modulation (HF power) by blue light than red and green lights [20]. To control and reduce the health risk of the use of blue-light-emitting device, further studies are required on the characteristics of the biological effects of blue light.

\section{Limitations}

This study has several limitations. First, the sample size was small and thus, the order effects of incidence angle may not be counterbalanced sufficiently. Second, there was only 10 -min dark period between the blue light exposures from different angles. This may have been too short to wash out the autonomic effects of preceding blue light from different incidence angles and may have resulted in an accumulation of the effect, although we have previously observed no significant autonomic effects 5 min after 30-min blue light exposure [22]. Third, we used OEL as blue light source. Although the spectral distributions of the light showed that the distribution mostly overlaps with the wavelength of melanopsin stimulating component, the results may not represent the features of general SSLs. Fourth, we performed the experiments during daytime. The results may differ with the time of day due to the possible influences of circadian rhythm. Finally, this study was designed to examine the acute autonomic effects of the incidence angle of blue light, but it can affect other non-image forming functions, such as melatonin secretion, circadian phase, sleep quality, and next morning alertness. To determine the long-term effects 
of incidence angle on these functions, research plans focused on effective irradiation angles are necessary. The present observations are thought to provide useful insights to such future studies.

\section{Declarations}

\section{Ethics approval and consent to participate}

All subjects in both studies gave their written informed consent to participate in this study. The study was performed according to the protocol approved by the Ethics Review Committee of Nagoya City University Graduate School of Medical Sciences and Nagoya City University Hospital (approved No. 60160164).

\section{Availability of data and material}

The datasets used and/or analyzed during the current study are available from the corresponding author on reasonable request.

\section{Funding}

This study was performed as a part of collaborative study with ENDO Lighting Co., Japan.

\section{Acknowledgements}

Not applicable.

\section{Consent for publication}

Not applicable.

\section{Competing interests}

The authors declare that they have no competing interests.

\section{Authors' contributions}

EY: concept/design, data analysis/interpretation, critical revision of article, and approval of article. YY: developed software, data collection, data analysis, and approval of article. NU: concept/design, critical revision of article, and approval of article. JH: concept/design, data interpretation, statistics, drafting article, and approval of article.

\section{Abbreviations}

ECG

electrocardiogram

$\mathrm{HF}$

high frequency 
HRV

heart rate variability

ipRGC

intrinsically photosensitive retinal ganglion cells

LED

light-emitting diode

LF

low frequency

OEL

organic electroluminescent

SDNN

standard deviation of R-R interval

SSL

solid-state lighting

\section{References}

1. Hatori M, Panda S. The emerging roles of melanopsin in behavioral adaptation to light. Trends Mol Med. 2010 Oct;16(10):435-46.

2. Hattar S, Liao HW, Takao M, Berson DM, Yau KW. Melanopsin-containing retinal ganglion cells: architecture, projections, and intrinsic photosensitivity. Science. 2002 Feb 8;295(5557):1065-70.

3. Berson DM, Dunn FA, Takao M. Phototransduction by retinal ganglion cells that set the circadian clock. Science. 2002 Feb 8;295(5557):1070-3.

4. Brainard GC, Hanifin JP. Photons, clocks, and consciousness. J Biol Rhythms. 2005 Aug;20(4):31425.

5. Kozaki T, Hidaka Y, Takakura JY, Kusano Y. Suppression of salivary melatonin secretion under 100$\mathrm{Hz}$ flickering and non-flickering blue light. Journal of physiological anthropology. 2018 Oct 19;37(1):23.

6. Katsuura T, Lee S. A review of the studies on nonvisual lighting effects in the field of physiological anthropology. Journal of physiological anthropology. 2019 Jan 22;38(1):2.

7. Yasukouchi A, Maeda T, Hara K, Furuune H. Non-visual effects of diurnal exposure to an artificial skylight, including nocturnal melatonin suppression. Journal of physiological anthropology. 2019 Aug 28;38(1):10.

8. Stevens RG, Brainard GC, Blask DE, Lockley SW, Motta ME. Adverse health effects of nighttime lighting: comments on American Medical Association policy statement. Am J Prev Med. 2013 Sep;45(3):343-6.

9. Stevens RG, Brainard GC, Blask DE, Lockley SW, Motta ME. Breast cancer and circadian disruption from electric lighting in the modern world. CA Cancer J Clin. 2014 May-Jun;64(3):207-18. 
10. Tosini G, Ferguson I, Tsubota K. Effects of blue light on the circadian system and eye physiology. Mol Vis. 2016;22:61-72.

11. Hatori M, Gronfier C, Van Gelder RN, Bernstein PS, Carreras J, Panda S, Marks F, Sliney D, Hunt CE, Hirota T, Furukawa T, Tsubota K. Global rise of potential health hazards caused by blue light-induced circadian disruption in modern aging societies. NPJ Aging Mech Dis. 2017;3:9.

12. Czeisler CA. Perspective: casting light on sleep deficiency. Nature. 2013 May 23;497(7450):S13.

13. Chang AM, Aeschbach D, Duffy JF, Czeisler CA. Evening use of light-emitting eReaders negatively affects sleep, circadian timing, and next-morning alertness. Proc Natl Acad Sci U S A. 2015 Jan 27;112(4):1232-7.

14. Yoshimura M, Kitazawa M, Maeda Y, Mimura M, Tsubota K, Kishimoto T. Smartphone viewing distance and sleep: an experimental study utilizing motion capture technology. Nature and science of sleep. 2017;9:59-65.

15. Lasko TA, Kripke DF, Elliot JA. Melatonin suppression by illumination of upper and lower visual fields. J Biol Rhythms. 1999 Apr;14(2):122-5.

16. Glickman G, Hanifin JP, Rollag MD, Wang J, Cooper H, Brainard GC. Inferior retinal light exposure is more effective than superior retinal exposure in suppressing melatonin in humans. J Biol Rhythms. 2003 Feb;18(1):71-9.

17. Price LLA. Report on the First International Workshop on Circadian and Neurophysiological Photometry, 2013. International Commission on Illumination, 2015 CIE TN 003:2015.

18. Enezi J, Revell V, Brown T, Wynne J, Schlangen L, Lucas R. A "melanopic" spectral efficiency function predicts the sensitivity of melanopsin photoreceptors to polychromatic lights. J Biol Rhythms. 2011 Aug;26(4):314-23.

19. Bailes HJ, Lucas RJ. Human melanopsin forms a pigment maximally sensitive to blue light (lambdamax approximately $479 \mathrm{~nm}$ ) supporting activation of $\mathrm{G}(\mathrm{q} / 11$ ) and $\mathrm{G}(\mathrm{i} / \mathrm{o})$ signalling cascades. Proceedings Biological sciences / The Royal Society. 2013 May 22;280(1759):20122987.

20. Yuda E, Ogasawara H, Yoshida Y, Hayano J. Suppression of vagal cardiac modulation by blue light in healthy subjects. Journal of physiological anthropology. 2016;35:24.

21. Yuda E, Ogasawara H, Yoshida Y, Hayano J. Enhancement of autonomic and psychomotor arousal by exposures to blue wavelength light: importance of both absolute and relative contents of melanopic component. Journal of physiological anthropology. 2017 Jan 31;36(1):13.

22. Yuda E, Ogasawara H, Yoshida Y, Hayano J. Exposure to blue light during lunch break: effects on autonomic arousal and behavioral alertness. Journal of physiological anthropology. $2017 \mathrm{Jul}$ 11;36(1):30. 
Figures

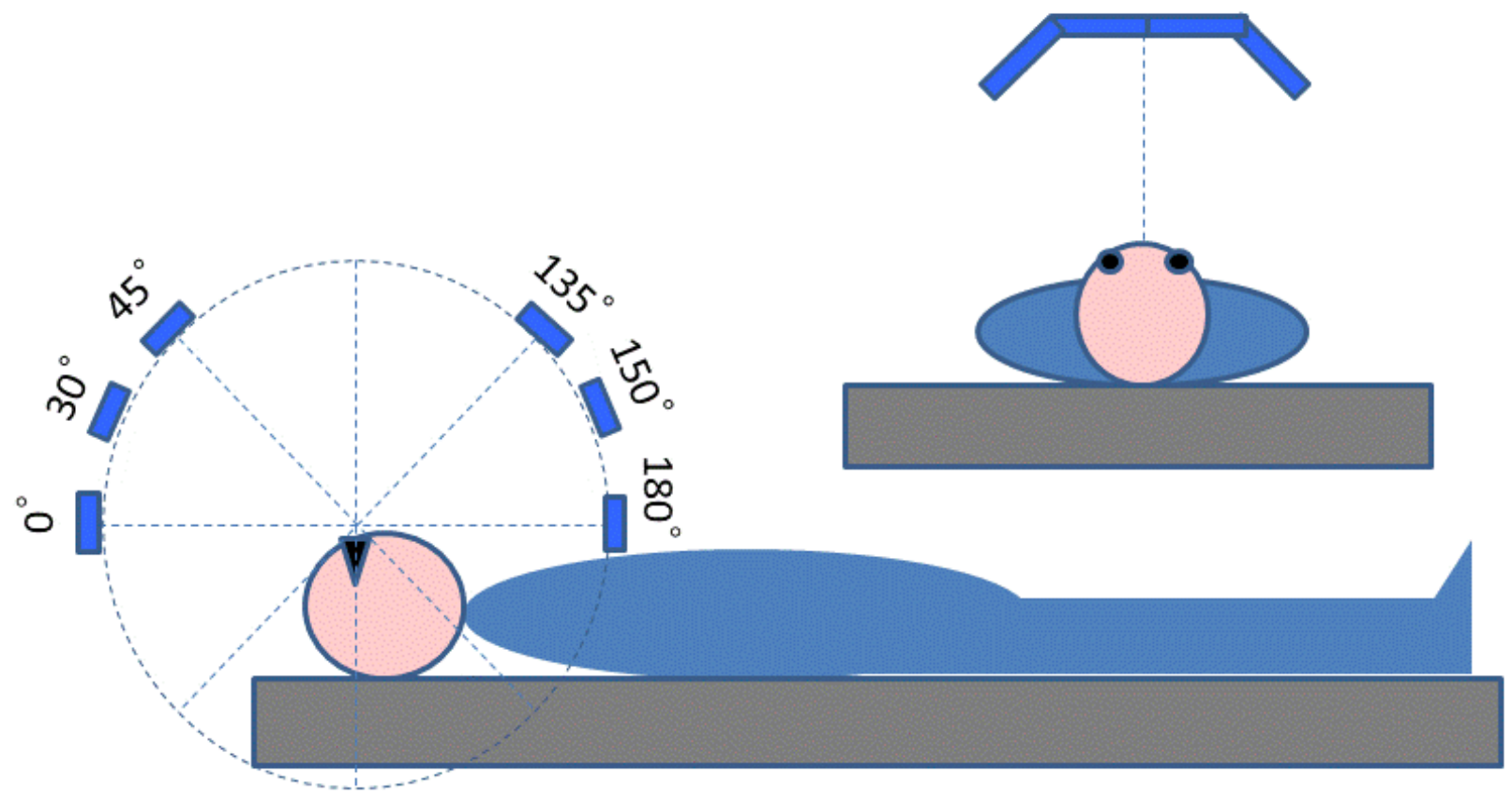

\section{Figure 1}

Schema of a lighting device for adjustable illumination angle The device can be moved in the sagittal plane of subject keeping the distance between the device and subject's eyes at $24 \mathrm{~cm}$. 


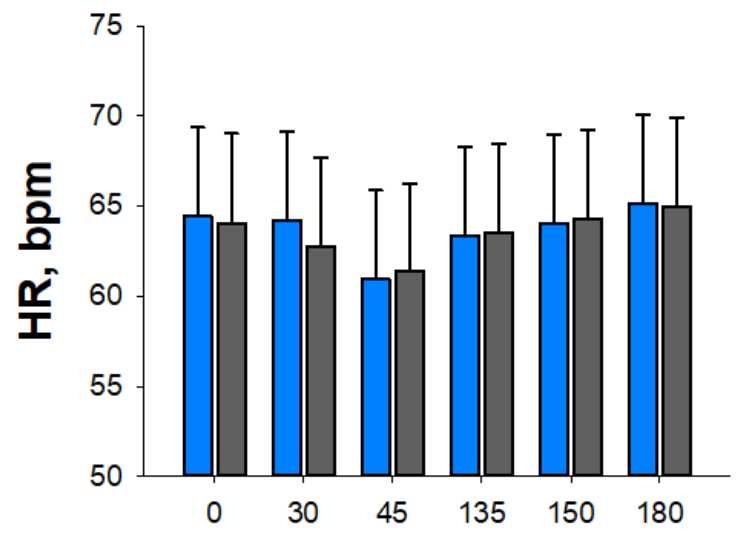

Incident angle

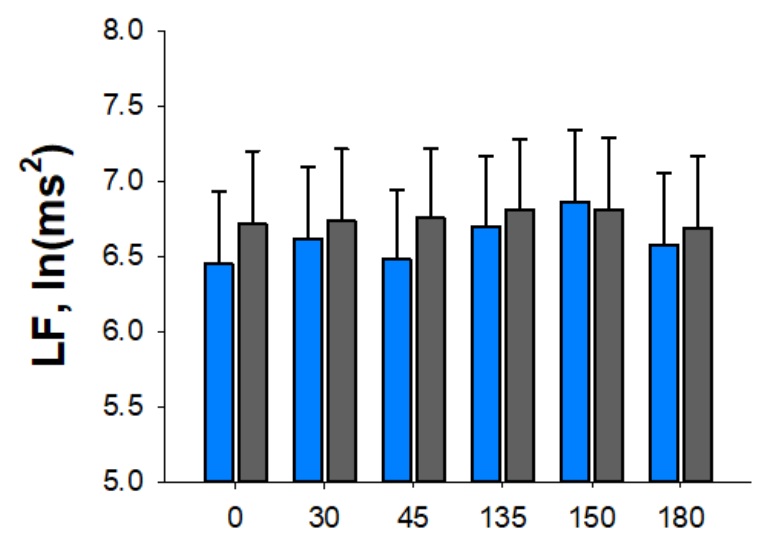

Incident angle

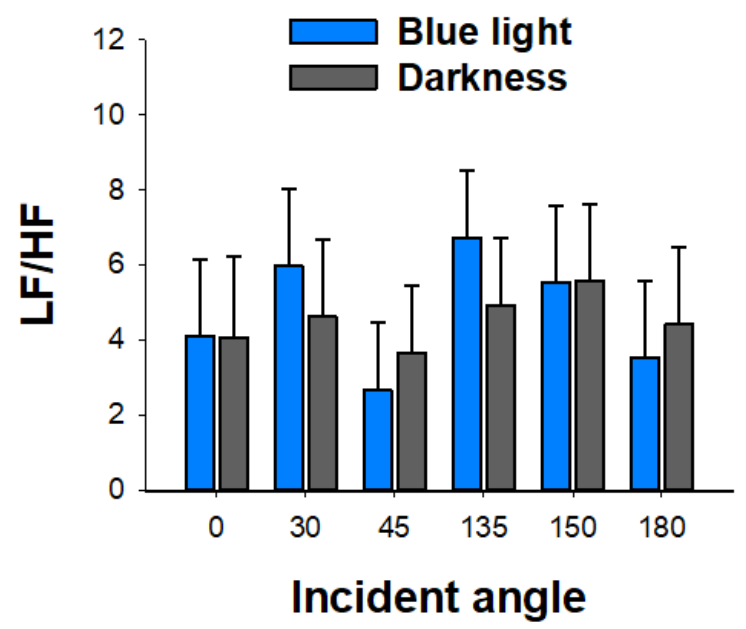

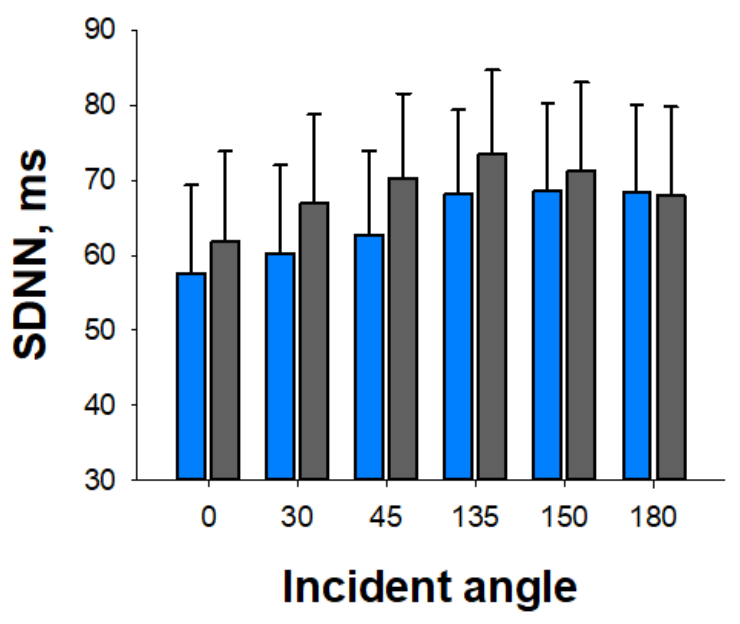
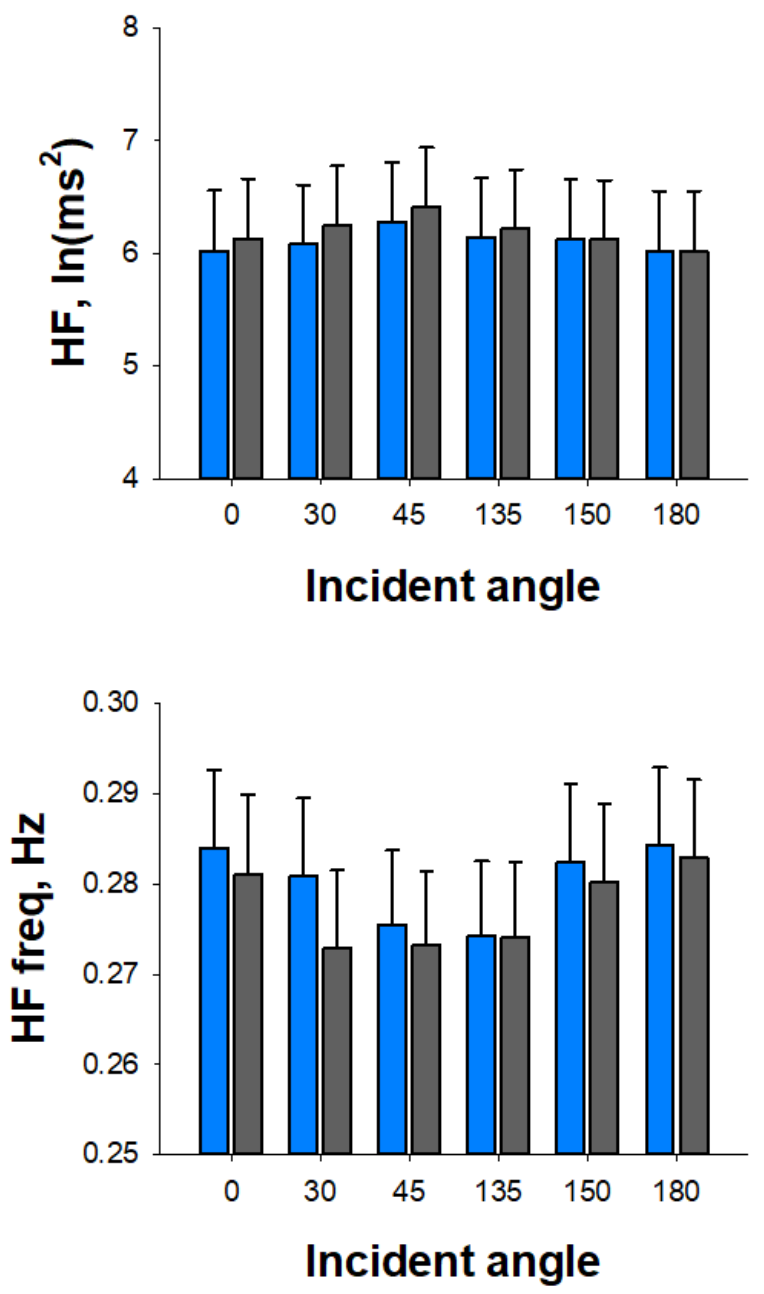

Figure 2

Heart rate (HR) and heart rate variability (HRV) indices during blue light exposure from different incidence angles on the eye (blue bars) and subsequent dark periods (gray bars). Values are least square means and standard errors of the means adjusted for the effects of exposure order. HF = high frequency, LF = low frequency, LF/HF = LF-to-HF power ratio, SDNN = standard deviation of normal-to-normal R-R intervals. 